\title{
ESTIMATIVA DA VIABILIDADE POLÍNICA DO MELÃO-DE-SÃO-CAETANO (Momordica charantia L.) COM DIFERENTES MÉTODOS DE COLORAÇÃO
}

Leila Pereira Neves Ramos ${ }^{1}$, Vera Lúcia Simões André ${ }^{1}$, Vanessa dos Santos de Mello $^{2}$, Fabiana Wink ${ }^{3}$, Isane Vera karsburg ${ }^{4}$

1 Graduada em Licenciatura Plena em Ciências Biológicas, pela Universidade do Estado de Mato Grosso, Campus de Alta Floresta, MT - Brasil

2 Bióloga, Mestranda no Programa de Pós Graduação em Genética e Melhoramento de Plantas, pela Universidade do Estado de Mato Grosso, campus de Alta Floresta, MT - Brasil

3 Graduanda do Curso de Bacharelado em Agronomia do Campus Universitário de Alta Floresta, MT - Brasil

4 Doutora em Genética e Melhoramento. Professora da Faculdade de Ciências

Biológicas e Agrárias. Universidade do Estado de Mato Grosso. 78.580-000 Alta Floresta, MT - Brasil.

e-mail de contato: nessa.demello@hotmail.com

Recebido em: 15/04/2017 - Aprovado em: 22/07/2017 - Publicado em: 31/07/2017 DOI: 10.18677/Agrarian_Academy_2017a23

\section{RESUMO}

Momordica charantia L. (Melão-de-São-Caetano) é uma espécie vegetal silvestre da família das cucurbitáceas, comumente encontrada em áreas urbanas e rurais, conhecida por possuir diversas propriedades medicinais. $O$ presente estudo objetivou estimar a viabilidade polínica de três populações de Momordica charantia L. ocorrentes em Alta Floresta - MT. Para este estudo foram utilizadas a solução de 2,3,5 trifeniltetrazolio (TTC) em duas concentrações: $0,075 \%$ e $0,30 \%$ expostos durante quatro tempos diferentes, e o corante Lugol $2 \%$. Para preparação das lâminas com o uso do teste trifeniltetrazolio (TTC), foi adicionada uma gota de cada tratamento sobre a lâmina, em seguida coberto com uma lamínula. Para o teste com Lugol $2 \%$, foi utilizada a técnica do esmagamento para a elaboração das laminas. $\mathrm{Na}$ estimativa da viabilidade polinica das populações 01,02 e 03 de $M$. charantia L., os testes colorimétricos (2,3,5 trifeniltetrazolio (TTC) e Lugol $2 \%$ ), foram capazes de distinguir os pólens viáveis dos inviáveis. Havendo maior viabilidade polínica da espécie em todas as populações em 12:00h para as concentrações de $0,075 \%$ e $0,30 \%$. A concentração de $0,30 \%$ mostrou-se eficiente em todas as populações e a concentração de $0,075 \%$, foi eficiente mesmo que a solução seja menos concentrada em comparação a solução de 0,30\%. Para os horários de 18:00h e 24:00h houve oscilação no processo de viabilidade polínica de $M$. charantia.

PALAVRAS-CHAVE: grãos de pólen, corantes, espécie medicinal, tetrazólio 


\title{
ESTIMATION VIABILITY MELÃO-DE-SÃO-CAETANO (Momordica charantia L.) BY METHODS COLORING
}

\begin{abstract}
Momordica charantia L. (Melon-de-São-Caetano) is a wild plant species of the cucurbit family, commonly found in urban and rural areas, known to have several medicinal properties. The present study aimed to estimate the pollen viability of three populations of Momordica charantia L. occurring in Alta Floresta-MT. For this study, the solution of 2,3,5-triphenyltetrazolium (TTC) was used in two concentrations: $0.075 \%$ and $0.30 \%$ exposed during four different times, and Lugol dye $2 \%$. To prepare the slides using the triphenyltetrazolium (TTC) test, one drop of each treatment was added to the slide, then covered with a cover slip. In the estimation of pollen viability of the populations 01,02 and 03 of $M$. charantia L., the colorimetric tests (2,3,5-triphenyltetrazolium TTC) and Lugol 2\%), were able to distinguish viable pollen from non-viable pollen. There was greater pollen viability of the species in all populations at 12 : $00 \mathrm{~h}$ for the $0.075 \%$ and $0.30 \%$ concentrations. The concentration of $0.30 \%$ was efficient in all populations and the concentration of $0.075 \%$ was efficient even though its solution was less concentrated compared to the $0.30 \%$ solution. For the hours of 18:00 and 24:00 there was oscillation in the process of pollen viability of $M$. charantia.
\end{abstract}

KEYWORDS: Pollen grains, medicinal species, dyes, tetrazolium

\section{INTRODUÇÃO}

O Melão-de-são-caetano (Momordica charantia L.), Cucurbitaceae, é uma trepadeira anual, sublenhosa, com caule longo e ramificado, cresce em média seis metros. Possui flores solitárias, de cor amarela com 2,5 a 3,5 cm. Fruto em formato de cápsula carnosa deiscente, fusiforme, com, 4 a $6 \mathrm{~cm}$ de comprimento na variedade, com costela longitudinal de papilas curtas, se abre quando maduros, em três valvas enroladas para fora, expondo as sementes envolvidas em um arilo vermelho, mucilaginoso e adocicado. É considerada uma planta daninha encontrada em pomares, cafezais, cercas, alambrados e em terrenos baldios (LORENZI, 2000).

Em relação à fitoterapia o $M$. charantia $\mathrm{L}$. é afrodisíaco, antidiabético, vermífugo e é utilizado no tratamento de reumatismo e diarreias (AGRA et al., 2007; SILVA et al., 2010; SOUSA et al., 2012). É uma planta comumente usada no tratamento e controle da diabete (NKAMBO et al., 2013). Por ser uma espécie que se destaca pelo potencial medicinal caseiro é necessária a realização de mais estudos referentes a espécie $M$. charantia (ASSIS et al., 2015).

A viabilidade polínica é um dos fatores responsáveis pela seleção de genótipos para programas de estudo e melhoramento das espécies (CABRAL et al., 2013). Portanto, entre as técnicas da citogenética, o estudo da viabilidade polínica é uma medida de fertilidade masculina, capaz de representar o grau de estabilidade dos genótipos. Quando a mesma é empregada em programas de melhoramento, fornece subsídios na tomada de decisão em programas de melhoramento da espécie (BRAMBATTI et al., 2016).

A utilização da solução de tetrazólio (TTC), tem sido considerada por muitos pesquisadores importante para otimização da aplicação dos recursos nos laboratórios, e possibilita a análise de amostras de espécies com menor custo mostrando-se eficiente quando colocado a teste (SILVA et al., 2013). O Lugol é utilizado em estudos como testes indicativos da viabilidade polínica (SOUZA et al., AGRARIAN ACADEMY, Centro Científico Conhecer - Goiânia, v.4, n.7; p.237 2017 
2002). Entretanto, este teste associa-se à detecção de substâncias constituintes dos grãos de pólen, como amido e os lipídios presentes no pólen (RODRIGUEZ-RIANO et al., 2000).

O objetivo desse trabalho foi estimar a viabilidade polínica de Momordica charantia L. por meio de testes colorimétricos, usando os corantes de 2,3,5 trifeniltetrazolio (TTC) em quatro tempos diferentes e duas temperaturas e Lugol $2 \%$, avaliando a eficiência dos corantes sobre a espécie testada.

\section{MATERIAL E MÉTODOS}

O trabalho foi realizado no Laboratório de Citogenética e Cultura de Tecidos Vegetais da Universidade do Estado de Mato Grosso, Campus de Alta Floresta - MT. Foram coletados 30 botões florais de Momordica charantia L. em estágio de préantese no município de Alta Floresta - MT para cada uma das três populações (Quadro 1).

QUADRO 1: Identificação dos acessos, local de coleta dos botões florais em préantese de Momordica charantia L. e dados de GPS.

\begin{tabular}{|c|c|c|}
\hline População & Local de Coleta & Coordenadas \\
\hline Pop.1 & Alta Floresta - MT & Sul: 0951'45,30 "Oeste: 56운'13,20" \\
\hline Pop. 2 & Alta Floresta - MT & Sul: 0951'14,40 "Oeste: $56^{\circ} 03^{\prime} 62,27^{\prime \prime}$ \\
\hline Pop. 3 & Alta Floresta - MT & Sul: $09^{\circ} 52^{\prime} 17,20$ "Oeste: $56^{\circ} 04^{\prime} 84,90 "$ \\
\hline
\end{tabular}

Para o teste de 2,3,5 trifeniltetrazolio (TTC), os botões florais foram cortados transversalmente com o auxílio de um bisturi, para a retirada das anteras, maceradas levemente com um bastão de vidro para a liberação dos grãos de pólen na solução de TTC em duas concentrações: $0,075 \%$ e $0,30 \%$, que permaneceram em quatro horários de exposição ao corante: 6:00, 12:00, 18:00 e 24:00 horas. 0 material foi conduzido em ambiente escuro com temperatura de $21^{\circ} \mathrm{C}$ e $30^{\circ} \mathrm{C}$.

Para a preparação das lâminas uma gota de cada tratamento foi depositada na lâmina, após o material foi coberto com uma lamínula. Para a análise das lâminas foi adotado o método de varredura, sendo contabilizados 300 grãos de pólen por lâmina com cinco repetições cada, totalizando 1500 grãos de pólen para cada tratamento, observadas sob microscópio óptico na objetiva de 40x. A viabilidade polínica foi determinada pela capacidade de coloração dos grãos de pólen, e foram considerados viáveis os que apresentaram coloração da exine e da intine ou protoplasma de vermelho e inviáveis aqueles que não apresentaram coloração.

$\mathrm{Na}$ estimativa da viabilidade polínica por meio do teste colorimétrico, foram utilizados botões florais em pré-antese, de uma população de $M$. charantia L. Foi testada a eficiência do corante, Lugol $2 \%$, para investigar a estimativa da viabilidade polínica da espécie. Para esse corante, a viabilidade foi determinada pela capacidade de coloração dos grãos de pólen, os quais foram considerados viáveis os pólens que apresentaram coloração da exina e da intina ou protoplasma bem definido e inviáveis aqueles que apresentaram exina corada e ausência de protoplasma.

No preparo das lâminas as anteras foram cortadas transversalmente com o auxílio de um bisturi. Para a liberação dos grãos de pólen, as anteras foram maceradas com um bastão de vidro, sobre uma gota de lugol $2 \%$. Após, o material foi coberto com uma lamínula e observado ao microscópio. A fim de obter uma 
amostragem ao acaso dos grãos de pólen corados, foi utilizado o método de varredura, sendo contabilizados 300 grãos de pólen por lâmina com cinco repetições cada, perfazendo um total de 1.500 grãos de pólen para o corante investigado. Todas as lâminas foram observadas no microscópio óptico na objetiva de $40 \mathrm{x}$ e as imagens foram digitalizadas utilizando o fotomicroscópio Leica, acoplado à câmera digital.

Com a contagem obtida de ambos os tratamentos, calculou-se a porcentagem de polens viáveis através da fórmula: № de grãos corados/ № de grãos contados * 100 e as médias do TTC comparadas pelo teste de Scott-Knott, e o Lugol 2\% pelo tukey, ambos em nível de $5 \%$ de probabilidade pelo programa $R$, versão 3.3 .2 ( $R$ CORE TEAM, 2016), com o auxílio do pacote ExpDes, versão 1.1.2 (FERREIRA et al., 2013).

\section{RESULTADOS E DISCUSSÃO}

$\mathrm{Na}$ avaliação da viabilidade polínica das três populações de Momordica charantia L. (Tabela 1), observou-se que as metodologias associadas às concentrações de 2,3,5 de cloreto de Trifeniltetrazólio (TTC), apresentaram diferenças significativas entre si. Os grãos de pólens deste estudo apresentaram taxas de viabilidade polínica consideradas baixa, média e alta. Conforme SOUZA et al., (2002), as duas concentrações de corantes nas técnicas utilizadas, apresentaram percentuais variáveis de $30 \%$ à $90 \%$.

TABELA 1. Percentuais da viabilidade dos grãos de pólen de Momordica charantia L., pela coloração com TTC em concentrações de 0,075\% e 0,30\% em duas diferentes temperaturas, e em quatro tempos de exposição.

\begin{tabular}{|c|c|c|c|c|c|c|}
\hline & Concentrarão & Temneratura 으 & & Tempc & (horas) & \\
\hline & Concentraçao & remperatura $=C$ & 6 & 12 & 18 & 24 \\
\hline & $0,075 \%$ & 21 & $38,56 b$ & $68,15 b$ & $60,89 b$ & $62,31 b$ \\
\hline & $0,30 \%$ & 21 & $49,85 b$ & $82,73 b$ & $75,57 b$ & $69,80 \mathrm{~b}$ \\
\hline & $0,075 \%$ & 30 & $49,64 a$ & $73,64 a$ & $59,55 b$ & $70,41 \mathrm{a}$ \\
\hline & $0,30 \%$ & 30 & $61,13 a$ & $95,96 \mathrm{a}$ & $92,42 \mathrm{a}$ & $91,16 \mathrm{a}$ \\
\hline & $0,075 \%$ & 21 & $39,27 b$ & $68,90 \mathrm{~b}$ & $60,69 b$ & $63,00 \mathrm{~b}$ \\
\hline & $0,30 \%$ & 21 & $51,37 b$ & $81,57 b$ & $75,26 b$ & $70,16 \mathrm{~b}$ \\
\hline & $0,075 \%$ & 30 & $51,24 a$ & $74,97 a$ & $58,82 b$ & $70,62 a$ \\
\hline & $0,30 \%$ & 30 & $62,18 \mathrm{a}$ & $96,76 a$ & $91,58 a$ & $90,51 a$ \\
\hline & $0,075 \%$ & 21 & $37,51 b$ & $69,52 b$ & $62,44 b$ & $63,11 b$ \\
\hline & $0,30 \%$ & 21 & $50,78 b$ & $83,67 b$ & $74,31 b$ & $71,04 b$ \\
\hline & $0,075 \%$ & 30 & $51,04 a$ & $75,46 a$ & $57,95 b$ & $71,86 a$ \\
\hline & & 30 & $61,42 a$ & $94,91 \mathrm{a}$ & $93,56 a$ & $91,71 \mathrm{a}$ \\
\hline
\end{tabular}

Médias seguidas pela mesma letra maiúscula nas linhas e minúscula nas colunas não diferem pelo teste de scott-knott a nível de 5\% de significância.

Destacou-se a população 3 (Tabela 1) com os maiores índices percentuais de pólens viáveis. Após a contagem dos pólens, foram feitos os cálculos para obtenção AGRARIAN ACADEMY, Centro Científico Conhecer - Goiânia, v.4, n.7; p.239 2017 
do percentual de viabilidade, de acordo com a coloração dos grãos de pólen. MUNHOZ et al. (2008), desenvolveram um trabalho de viabilidade polínica com a espécie Carica papaya L., com o teste de Tetrazólio (TTC) que mostrou-se como uma ferramenta confiável com estimativa rápida de viabilidade, fornecendo estimativa de viabilidade polínica de $67,5 \%$.

Para o corante utilizado (Lugol $2 \%$ ), a espécie de $M$. charantia, apresentou uma alta taxa de pólens viáveis, sendo a média geral do corante utilizado para as três Populações da espécie em estudo, superior a $80 \%$, não se diferenciando entre si estatisticamente (Tabela 2).

SANTOS et al. (2015), obtiveram resultados com altas médias de viabilidade utilizando o corante Lugol, o qual apresentou média de $98,83 \%$, seguido do corante Alexander (98,19\%) e do corante Carmim Acético (95,11\%), recomendando o uso de qualquer um dos dois primeiros corantes citados, em testes de viabilidade com a espécie $B$. excelsa, uma vez que as médias foram maiores. Segundo DIEGUES et al. (2015) em um trabalho feito com a espécie Jatropha curcas L. a menor taxa de viabilidade polínica foi de $78 \%$, enquanto que a máxima observada foi de $96 \%$. Com base nos resultados obtidos, apesar de $J$. curcas ter apresentado certas anomalias durante a meiose, os acessos analisados mostraram viabilidade polínica satisfatória para o material estudado.

TABELA 2. Médias percentuais de viabilidade do pólen entre as populações de Momordica charantia L., estimada pelo corante Lugol $2 \%$.

\begin{tabular}{cc}
\hline Populações & Viabilidade polínica (\%) \\
\hline $\mathbf{0 1}$ & $82,96 \mathrm{a}$ \\
$\mathbf{0 2}$ & $85,33 \mathrm{a}$ \\
$\mathbf{0 3}$ & $80,83 \mathrm{a}$ \\
\hline $\mathbf{C V}(\%)$ & 14,03 \\
\hline
\end{tabular}

Médias seguidas pela mesma letra minúscula na coluna não diferem pelo teste de tukey a nível de $5 \%$ de significância.

Observa-se nas Figuras 1 e 2, no estudo com as populações 01 (A), 02 (B) e $03(\mathrm{C})$, o horário em que houve maior viabilidade polínica dos pólens da espécie de $M$. charantia $\mathrm{L}$. em ambiente com temperatura controlada à $21^{\circ} \mathrm{C}$ e $30^{\circ} \mathrm{C}$ foi $\mathrm{o}$ horário de 12:00h em tempo de exposição, tanto para a concentração do tetrazólio (TTC) de 0,075\% como também para o de 0,30\%. Destacando-se a eficiência da concentração de $0,30 \%$ em todas as populações e a importância do uso da concentração do tetrazólio $0,075 \%$, pois o mesmo mostrou-se eficiente mesmo tendo a solução menos concentrada em comparação ao de $0,30 \%$. Para os horários de 18:00h e 24:00h em ambas populações constatou-se que houve oscilação do processo de viabilidade dos pólens de $M$. charantia $L$.

De acordo com SANTOS et al. (2016), em Costus spiralis (Jacq.) Roscoe, obtiveram resultados semelhantes ao deste estudo, que verificaram que a concentração de $0,30 \%$ com 12 horas de exposição obteve coloração vermelho intenso os grãos de pólen viáveis. E ainda segundo os mesmos autores o teste do tetrazólio (TTC) é considerado confiável permitindo a distinção entre os pólens viáveis por apresentar a reação química e os não viáveis com ausência de coloração, sendo um corante relacionado com a respiração celular do pólen, estando corado apenas os que se encontram vivos ou viáveis. LEITE et al. (2016), estimaram a viabilidade polínica de Jacaranda copaia (Aubl.) D. Don., utilizando as 
concentrações de $0,075 \%$ e $0,30 \%$ em diferentes tempos de exposição, para esta espécie o tratamento que melhor representou a viabilidade foi com $0,30 \%$ de concentração por 12:00h caso haja necessidade do teste rápido, mas, caso a avaliação seja mais precisa pode utilizar a concentração de $0,075 \%$ por $24: 00 \mathrm{~h}$, sendo esta concentração mais eficiente apresentando maior porcentagem de pólen viáveis, caso não seja necessário uma resposta rápida.

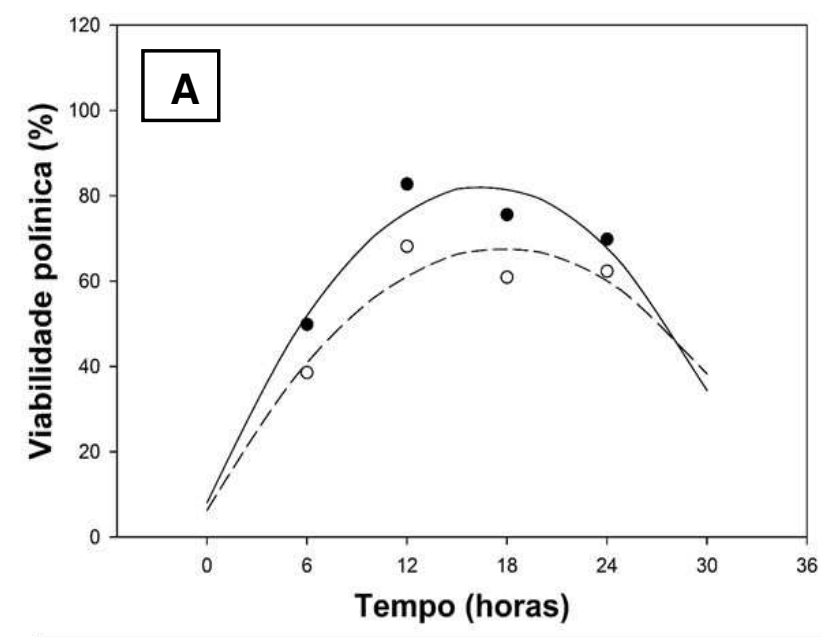

- $\quad$ TTC $0,30 \%$

$y=8,0170000+8,9289667^{*}$ tempo- $0,2683611^{\star}$ tempo ${ }^{2} R^{2}=0,85$ - TTC $0,075 \%$

$---y=6,2610000+6,9366000 *$ tempo-0,1956667*tempo ${ }^{2} R^{2}=0,79$

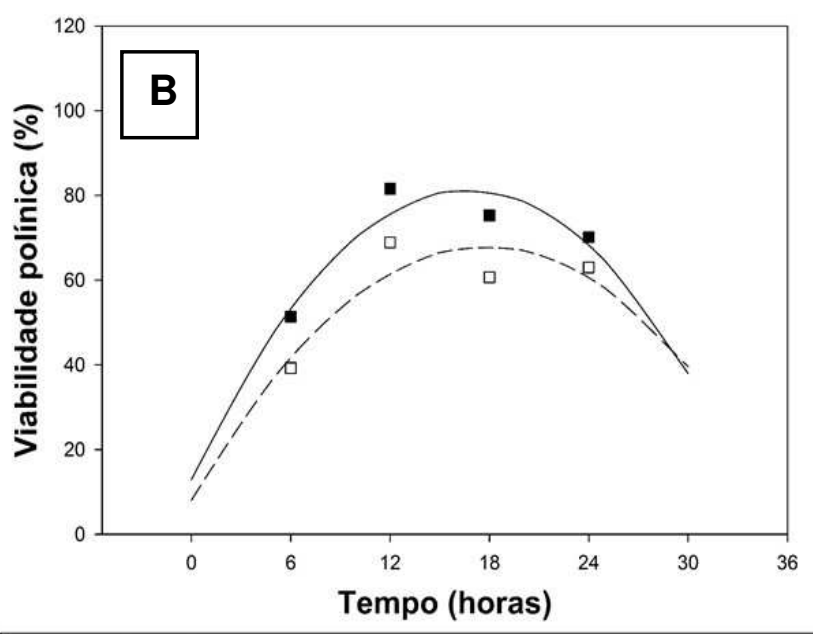

- TTC $0,30 \%$

$y=12,9459999+8,1900333^{\star}$ tempo- $0,2451944^{\star}$ tempo $^{2} R^{2}=0,85$ a TTC $0,075 \%$

- - $y=8,0615000+6,7433167^{*}$ tempo- $0,1897917^{*}$ tempo ${ }^{2} R^{2}=0,76$

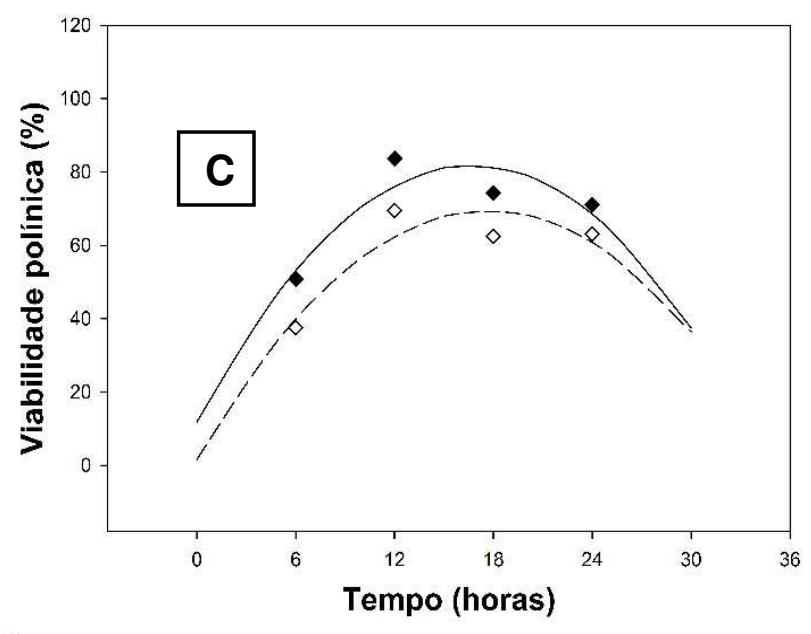

\footnotetext{
- TTC $0,30 \%$

$y=11,8995000+8,3894500^{*}$ tempo-0,2510694*tempo ${ }^{2} \quad \mathrm{R}^{2}=0,79$ TTC $0,075 \%$

$y=1,5375000+7,6925167^{*}$ tempo- $0,2176806^{*}$ tempo ${ }^{2} \quad R^{2}=0,81$
}

FIGURA 1. Viabilidade polínica de populações de Momordica charantia L. estimada com o uso de concentrações de tetrazólio em função do tempo a $21 \stackrel{\circ}{\circ}$. (A) População 01; (B) População 02 e (C) População 03. 


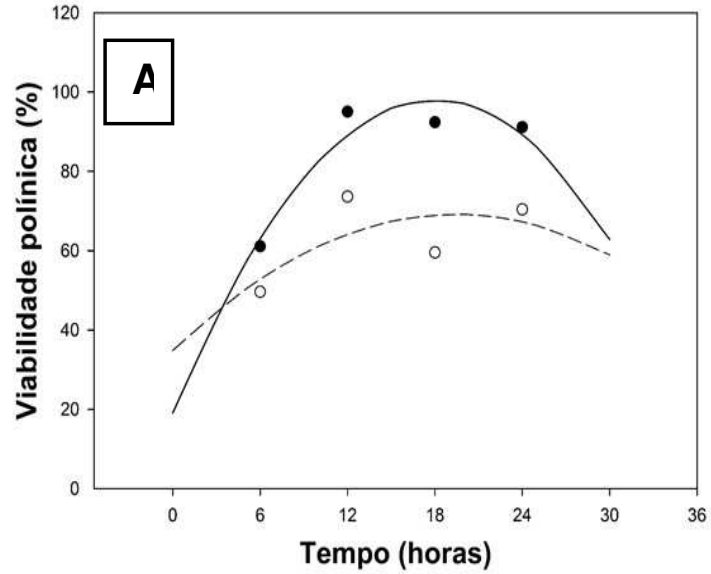

- $\quad$ TTC $0,30 \%$

$y=19,1050000+8,7879000^{*}$ tempo- $0,2443611^{*}$ tempo ${ }^{2} R^{2}=0,90$ TTC $0,075 \%$ $y=34,83600000+3,54060000^{*}$ tempo- $0,09122222^{*}$ tempo ${ }^{2} \quad R^{2}=0,44$

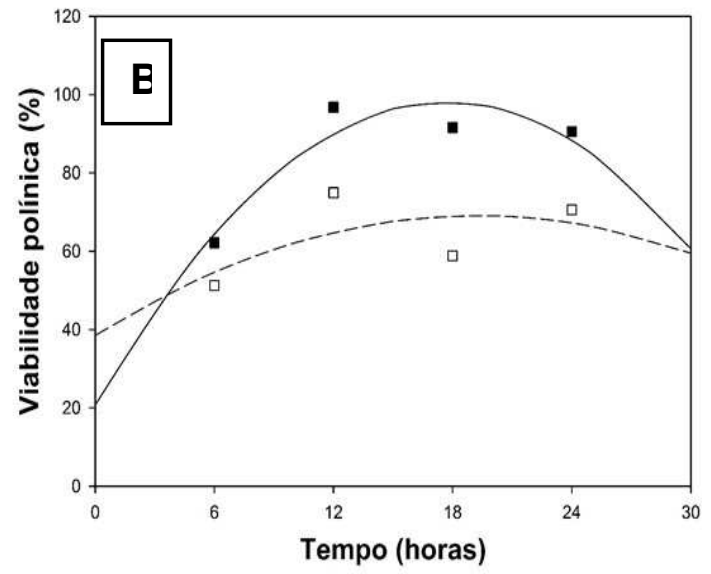

TTC $0,30 \%$

$y=20,747000+8,7562333^{\star}$ tempo- $0,2475278^{\star}$ tempo $^{2} R^{2}=0,86$ TTC $0,075 \%$

$y=38,51300000+3,18446667^{\star}$ tempo- $0,08283333^{\star}$ tempo ${ }^{2} R^{2}=0,34$

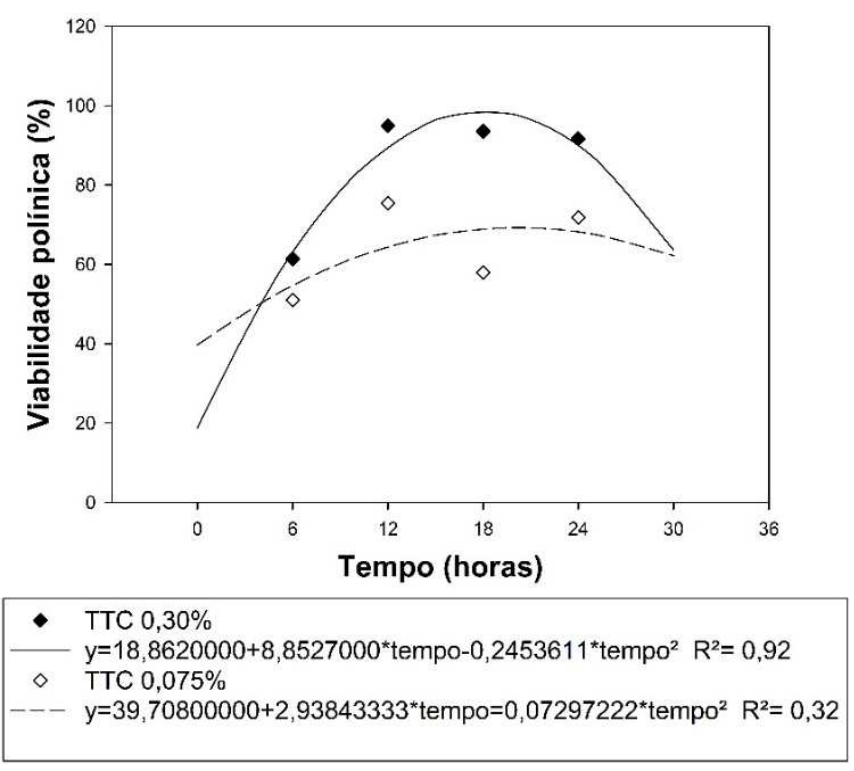

FIGURA 2. Viabilidade polínica de populações de Momordica charantia L. estimada com o uso de concentrações de tetrazólio em função do tempo a 30 ㄷ. (A) População 01; (B) População 02 e (C) População 03.

\section{CONCLUSÃO}

Os testes colorimétricos 2,3,5 trifeniltetrazolio (TTC) e Lugol 2\%, mostraramse eficientes para a diferenciação dos pólens viáveis dos inviáveis, apresentando médias altas de viabilidade, assim, ambos podem ser indicados para estimar a viabilidade polínica de Momordica charantia L. Com a utilização do teste de TTC recomenda-se utilizar a concentração de $0,075 \%$ em temperatura de $30{ }^{\circ} \mathrm{C}$ durante 12 horas de exposição dos grãos de pólen a solução. 


\section{AGRADECIMENTOS}

Os autores agradecem à Universidade do Estado de Mato Grosso, (UNEMAT) Alta Floresta - MT.

\section{REFERÊNCIAS}

AGRA, M. de F.; FREITAS, P. F. de.; BARBOSA-FILHO, J. M. Synopsis of the plants known as medicinal and poisonous in Northeast of Brazil. Revista Brasileira de Farmacognosia, v. 17, n. 1, p. 114-140, 2007. Disponível em: <http://dx.doi.org/10.1590/S0102-695X2007000100021>. doi: 10.1590/S0102-695X2007000100021

ASSIS, J.; SOUSA, R.; LINHARES, P. F.; PEREIRA, M.; MOREIRA, J. Avaliação biométrica de caracteres do melão de São Caetano (Momordica charantia L). Revista brasileira de plantas medicinas, v. 17, n. 4, p. 505-514, 2015. Disponível em: <http://www.scielo.br/pdf/rbpm/v17n4/1516-0572-rbpm-17-40505.pdf>. doi: $10.1590 / 1983-084 X / 13062$

BRAMBATTI, A.; BRAMMER, S. P.; WIETHÖLTER, P.; JUNIOR, A. do. N. Estabilidade genética em triticale estimada pela viabilidade polínica. Arquivos do Instituto Biológico, v. 83, p. 01-07, 2016. Disponível em:

<http://dx.doi.org/10.1590/1808-1657000802014>.doi.org/10.1590/18081657000802014

CABRAL, J. C.; ROSSI, A. A. B.; KLEIN, M. E.; VIEIRA, F. S.; GIUSTINA, L. D. Estimativa da viabilidade polínica em acessos de Theobroma cacao L. baseada em testes colorimétricos. Enciclopédia Biosfera, v.9, n.17, p.2780-2788, 2013. Disponível em: <http://www.conhecer.org.br/enciclop/2013b/CIENCIAS\%20BIOLOGICAS/estimativa \%20da\%20viabilidade.pdf>.

DIEGUES, P. I.; JUNIOR, P. C. D.; RIBEIRO, N. V. da. S.; REIS, M. V. M.; ABBOUD, A. C. de. S. Comportamento meiótico e viabilidade polínica na espécie Jatropha curcas L.Ciências Agrárias, v. 36, n. 1, 2015.Disponível em:

<http://www.uel.br/revistas/uel/index.php/semagrarias/article/viewFile/14926/16036>. doi: 10.5433/1679-0359.2015v36n1p141

FERREIRA, E. B.; CAVALCANTI, P. P.; NOGUEIRA, D. A. ExpDes: Experimental Designs package. $R$ package version 1.1.2, 2013.

LORENZI, H. Plantas Daninhas do Brasil: terrestres, aquáticas, parasitas e tóxicas. 3.ed. Nova Odessa: Instituto Plantarum - SP, 2000. 640 p.

LEITE, D. M.; SANTOS, B. N. V. dos.; SANTOS, L. C. B. dos., DAMASIO, J. F.; MELLO, V. D. S. de.; ALMEIDA, M. W. de.; KARSBURG, I. V. (2016). Avaliação da viabilidade polínica de Pará-Pará com uso de Trifenil cloreto de Tetrazolio. Ciência \& Tecnologia: Fatec-JB, Jaboticabal, v. 8, n.1, 2016.

Disponível em: <www.citec.fatecjab.edu.br/index.php/files/article/view/998>. 
MUNHOZ, M. Á. R. C. I. A.; LUZ, C. F. P.; MEISSNER, F. P. E.; BARTH, O. M.; REINERT, F. Viabilidade polínica de Carica papaya L.: uma comparação metodológica. Revista Brasileira de Botânica, v. 31, n. 2, p. 209-214, 2008.

Disponível em: <http://www.scielo.br/pdf/rbb/v31n2/v31n2a03.pdf>.

NKAMBO, W.; ANYAMA, N. G.; ONEGI, B. In vivo hypoglycemic effect of methanolic fruit extract of Momordica charantia L. African health sciences, v. 13, n. 4, p. 933939, 2013.Disponível em: $<$ https://www.ncbi.nlm.nih.gov/pmc/articles/PMC4056504/>. doi: 10.4314/ahs.v13i4.11

R Core Team. R: A language and environment for statistical computing. $R$ Foundation for Statistical Computing, Vienna, Austria. URL https://www.R project.org/. 2016.

RODRIGUEZ-RIANO, T.; DAFNI, A. A new procedure to asses pollen viability. Sexual Plant Reproduction, v. 12, n. 4, p. 241-244, 2000.Disponível em: <https://link.springer.com/article/10.1007/s004970050008>.doi: $\quad 10.1007$ s004970050008

SANTOS, B. N. V. dos.; ALMEIDA, M. W. de.; MELLO, V. D. S. de.; DAMASIO, J. F.; SANTOS, L. C. B. dos.; LEITE, D. M.; KARSBURG, I. V. Estimativa da viabilidade polínica de cana-de-macaco baseado no uso de 2, 3, 5 cloreto de Trifeniltetrazolio. Ciência \& Tecnologia: Fatec-JB, Jaboticabal, v. 8, n.1, 2016. Disponível em: $<$ http://www.citec.fatecjab.edu.br/index.php/files/article/download/991/pdf>.

SANTOS, T. A. dos.; TIAGO, P. V.; SCHMITT, K. F. M.; MARTINS , K. C.; ROSSI, A.A. B. Viabilidade pólinica em Bertholletia excelsa Bonpl. (LECYTHIDACEAE) baseada em diferentes testes colorimétricos. Enciclopédia Biosfera, Centro Científico Conhecer - Goiânia, v.11 n.22; p. 2015. Disponível em: $<$ http://dx.doi.org/10.18677/Enciclopedia_Biosfera_2015_030>. doi: org/10.18677/Enciclopedia_Biosfera_2015_030

SILVA, M. A.; BARBOSA, J. S.; ALBUQUERQUE, H. N. Levantamento das plantas espontâneas e suas potencialidades fitoterapêuticas: um estudo no complexo Aluízio Campos-Campina Grande-PB. Revista Brasileira de Informações Científicas, v. 1, n. 1, p. 52-66, 2010. Disponível em: <http://www.rbic.com.br/artigos\%20pdf/vol1_n1\%20-\%202010/7_vol1rbic.pdf>.

SILVA, R. C. de.; SOUZA, G, C. R. de.; de BARROS, F. N., J.; PANOBIANCO, M. Adaptação do teste de tetrazólio para avaliação da viabilidade e do vigor de sementes de girassol. Pesquisa Agropecuária Brasileira, v. 48, n. 1, p. 105-113, 2013. Disponível em: <http://www.scielo.br/pdf/pab/v48n1/14.pdf>. doi: 10.1590/S0100-204X2013000100014

SOUSA, F. F.; SANTOS, A. L. dos.; ANDRADE, L. O.; QUEIROZ, M. F. Identificação de plantas espontâneas com propriedades terapêuticas em área 
cultivada com jatropha sp. Revista Verde de Agroecologia e Desenvolvimento Sustentável, v. 6, n. 4, p. 258-262, 2012. Disponível em:

$<$ http://revistas.ufcg.edu.br/acsa/index.php/ACSA/article/viewFile/651/pdf>.

SOUZA, M. M.; PEREIRA, T. N. S.; MARTINS, E. R. Microsporogênese e microgametogênese associadas ao tamanho do botão floral e da antera e viabilidade polínica em maracujazeiro-amarelo (Passiflora edulis sims f. flavicarpa degener). Lavras. Ciência e agrotecnologia. 26: 1209-1217.2002.

Disponível em:

<http://www.scielo.br/scielo.php?script=sci_nlinks\&ref=000152\&pid=S1807$8621201400020001200023 \& \operatorname{lng}=\mathrm{en}>$. 